\title{
Current progress of China's free ART program
}

\author{
Fu Jie ZHANG*, Jennifer PAN, Lan YU, Yi WEN, Yan ZHAO \\ Division of Treatment and Care, National Center for STD/AIDS Prevention and Control, Chinese Center for Disease Preven- \\ tion and Control, Beijing 100050, China
}

\begin{abstract}
China's Free ART Program was initiated in 2002 as an emergency response to save and improve the lives of AIDS patients living mainly in impoverished rural regions of central China. With little experience in HIV/AIDS treatment and care and resource limitations, China's efforts to provide widespread access to free antiretroviral therapy has been a process fraught with difficulty. However, the Free ART Program is progressing from an emergency response to a standardized treatment and care system. The development of national guidelines, training programs, a laboratory support network, a national patient database, programs for special populations such as children and patients living with coinfections, and operational research has improved the scope and quality of the free treatment program. As of June 30, 2005, a total of 19,456 patients in 28 provinces, autonomous regions, and special municipalities had received free ART. Challenges stemming from the nature of China's health system and patient population persist, but with strong government support and a diverse set of resources, China has the capacity to overcome these challenges and to provide nationwide access to high quality treatment and care.
\end{abstract}

Keywords: human immunodeficiency virus, acquired immune deficiency syndrome, China, antiretroviral therapy, free ART program, treatment and care.

\section{INTRODUCTION}

At the end of 2003, there were an estimated 840,000 people in China infected with HIV, among whom 80,000 were in need of antiretroviral therapy (ART) [1]. Since 1999 , the annual rate of increase in reported HIV infections has been around $30 \%$, with growing numbers of females becoming infected [1]. The transmission of HIV/ AIDS in China has been through intravenous drug use (43. $2 \%$ ) in the south, southwest, and western provinces; prior commercial blood and plasma selling $(26.8 \%)$ in central China; and sexual intercourse (8.3\%) in major cities, especially in the south $[2,3]$. Blood plasma selling activities in Henan province from 1994-1995 created a unique pattern in the spread of the disease, namely a short timeframe of transmission and geographical clustering, leading to the rapid emergence of a large number of AIDS cases in the early 2000 's. It was critical for the government to re-

\footnotetext{
*Correspondence: Fu Jie ZHANG

Tel: 86-10-6303-9086; Fax: 86-10-6303-9087;

E-mail: treatment@chinaaids.org.cn
}

spond expeditiously to these impoverished patients who were in desperate need of ART but did not have the financial capability to acquire such treatments. Therefore, the distribution of ARV was initiated despite the lack of a comprehensive care infrastructure for managing AIDS and opportunistic infections (OIs).

In December 2001, the Division of Treatment and Care (Division) was created within the National Center for STD/ AIDS Prevention and Control (NCAIDS) and the Chinese Centers for Disease Control (CDC). Less than a year later, with technical assistance from the Division, the first free ART program was piloted in Shangcai county, a county in southern Henan province beset by plasma selling activities in the 1990s. Physicians were trained in the field, and one hundred patients were placed on a combination of domestic and imported ARVs. At the end of this year, the government issued a set of policies to increase access to ARVs such as waiving tariffs and value added taxes for imported ARVs and creating a fast track for domestic ARV approval by the State Food and Drug Administration.

Following the success of the Shangcai county pilot, the government decided to scale up free treatment in early 
2003 through the China Comprehensive Response Program (China CARES), which spanned a number of high prevalence counties. China CARES counties received 300, 000 to $600,000 \mathrm{CNY}(\$ 37,000-\$ 74,000)$ in matching funds from the central and local government, augmenting an increasing general HIV/AIDS budget of 300 million CNY (\$37 million) in 2002, 470 million CNY (\$58 million) in 2003 , and 810 million CNY ( $\$ 100$ million) in 2004. By the end of 2003, seven thousands people had received free ART.

Due to the urgent nature of patients' needs and the need for a quick government response, the initial 20022003 phase of China's free treatment and care efforts were managed and implemented by the disease control system, led at the national level by the Ministry of Health (MOH) Department of Disease Control and NCAIDS, and by local CDCs at the provincial, prefectural, and county levels. ARVs were distributed through the CDC system, and patients were seen at CDC clinics or township hospitals and village clinics overseen by the CDC. Resource limitations and system constraints resulted in vast variations between different localities in OIs management, conduct of laboratory testing, and patient support. During this initiation phase, field training and treatment guidelines were provided by NCAIDS and a three month reporting system was developed to monitor patient enrollment.

Many challenges were encountered during this initial phase, but they provided valuable lessons and helped build the foundation for the national Free ART Program. At the end of 2003, Premier Jia Bao WEN announced the "Four Frees and One Care" policy during his visit to AIDS patients at Beijing Ditan Hospital. The first tenant of this policy states that free ARV drugs will be given to all rural AIDS patients and urban AIDS patients facing financial difficulties [2]. To assist the implementation of the Free ART Program as mandated in the "Four Frees and One Care" policy, two subsequent policy documents, the "Measures for Management of Drug Treatment of HIV/AIDS and Common OIs at No/Reduced Charge" ("Measures") [4] and the "Opinions Concerning the Management of HIV Antiviral Treatment," ("Opinions") [5] were issued.

"Measures" delineates the roles of different institutional actors and broadens the scope of responsibility for treatment and care from the CDC system to the hospital system. The central government takes on the costs of ARV drugs, Prevention of Mother to Child Transmission (PMTCT) drugs, and reagents for infant testing. The local government assumes all other costs, including those for OI drugs and eliminating barriers to accessing treatment. Measures describe drug management procedures and it includes a framework for monitoring and evaluation (M\&E), as well as a directory of free ARV drugs and a list of common
HIV-related OIs. "Opinions" delineates responsibilities for the management and implementation of the Free ART Program at the national, provincial, prefectural, and county levels. At the national level, the $\mathrm{MOH}$ is responsible for the overall management and supervision of treatment and care, including the creation of an expert panel, the National HIV/ AIDS Clinical Taskforce, to develop guidelines and technical documents, to train provincial health workers, and to provide technical guidance. At the provincial level, bureaus of health are responsible for managing treatment within the province, including the creation of provincial expert panels and the appointment of hospitals to treat patients with critical illnesses, serious OIs, other comorbidities and complications. At the prefectural and county level, "Opinions" stipulates that expert panels should be formed to provide guidance for diagnosis; assessment of treatment efficacy; management of serious OIs, adverse reactions, and complications, and supervision patient follow-up at the township, village, and community levels. At the prefectural level, hospitals should be designated to provide health counseling, antenatal and delivery services for HIV positive pregnant women, and regular follow-up and monitoring of patients. CDCs at both the county and prefectural level are responsible for coordinating home-based treatment, which is the basic principle of ART in China. $\mathrm{CDCs}$ are also responsible for the collection and reporting of patient data and the management and distribution of ARV drugs. County and prefectural clinicians are responsible for providing follow-up diagnosis, routine examinations of patients receiving ART, and fine tuning of treatment regimens.

With the groundwork laid by initial pilots and policy developments, China's treatment and care efforts have been progressing from an emergency response to a standardized system. The Chinese government has identified treatment and care as an urgent issue, and substantial resources have been devoted to these efforts. A multitude of challenges, rooted in the rural nature of the epidemic and the lack of human and technical resources amplify the complexity of treatment and care. However, with the lessons from the initial phase of treatment and increasing government involvement, China has the ability to address emerging challenges and to improve the Free ART Program.

\section{CURRENT PROGRESS OF TREATMENT AND CARE IN CHINA}

\section{Coverage}

As of June 30, 2005, a cumulative of 19,456 patients had received free ART, with an $8 \%$ dropout rate and a 10 . $2 \%$ death rate [6]. These patients live in 441 counties in 28 of China's provinces, autonomous regions, and special municipalities [6]. Data from 2004 shows that nearly $95 \%$ of 
deaths were caused by HIV-related diseases, the majority occurring within the first three months of treatment [7]. Slightly over $60 \%$ of dropouts also occurred within the first three month, and data from June 2005 shows that the most common reasons for discontinuing treatment were "other" side effects, patient request, difficulty with adherence, and progression of disease, in that order [6].

\section{Treatment regimen and criteria}

China's recommended first line regimen is the World Health Organization (WHO) regimen of zidovudine (AZT) or stavudine $(\mathrm{d} 4 \mathrm{~T})+$ lamivudine $(3 \mathrm{TC})+$ nevirapine (NVP). This regimen, however, did not become available until 2005 when 3 TC first entered the Chinese market. As a result, approximately $80 \%$ of patients currently receiving ART are on the initial treatment regimen of AZT or $\mathrm{d} 4 \mathrm{~T}+$ (didanosine) ddI + NVP. Also available to patients receiving free treatment are indinavir (IDV) and efavirenz (EFV). The eligibility criteria for ART is predicated on confirmation of HIV seropositivity and is divided into clinical criteria of the WHO Stage III or IV, symptomatic disease, or extrapulmonary TB or laboratory criteria of CD4 count below 200 cells $/ \mathrm{mm}^{3}$ and in the absence of CD4, total lymphocyte count below 1200 cells $/ \mathrm{mm}^{3}$ [8].

\section{Guidelines}

The MOH National HIV/AIDS Clinical Taskforce, consisting of twenty-five of China's most experienced HIV/ AIDS physicians, CDC personnel, and virologists, is responsible for guideline development and training. In December of 2004, with support from various international organizations, the taskforce developed the "China Free ARV Manual." This manual stipulates the standard requirements for implementing the Free ART Program as well as treatment strategy, entry criteria and preparation, ARV treatment, follow-up, management of side effects, treatment failure, adherence, and other treatment support procedures. Other materials developed by the taskforce include the "HIV/AIDS Clinical Treatment and Nursing Training Curriculum, 2003," and in conjunction with the Chinese Medical Association, the "Guidelines for AIDS Diagnosis and Treatment."

\section{Training}

Although Measures gives local governments the responsibility of training health workers, the central government realized that it would take time to develop local training capacity. The MOH therefore tasked the Division of Treatment and Care to design and implement national level training courses for provincial and county level health workers. Two types of training are currently conducted by the Division with technical support from the HIV/AIDS Clinical
Taskforce. The first, a didactic five-day training course targeting provincial physicians with more training and experience, has been provided to over 700 physicians. The second, a two-month mini residency course mainly targeting county level physicians, has covered approximately 520 physicians. Two-month courses are held at nine urban hospitals located throughout China, designated by the $\mathrm{MOH}$ as national HIV/AIDS training centers. The Division in conjunction with US CDC and the Clinton Foundation has also set up a rural training center in Anhui province for county-level physicians and staffed by a U.S. trained HIV/ AIDS physician. Web-based education and a Voluntary Counseling and Testing (VCT) training course are also available. With support from the Clinton Foundation, physicians have been sent abroad to receive clinical training at U.S. universities.

The national training curriculum emphasizes the adaptation of materials to the appropriate level of the provider. To supplement this curriculum, the division also collects Chinese-language training materials from other organizations conducting training. Some of these other training programs include the MOH and Yale-China nurse training program and regional physician training by Project Hope. In addition to national level government programs, provincial and local governments have also conducted their own training programs. In order to assist these local programs, the Division is developing a national database of annually updated training needs and resources.

\section{Laboratory}

Responsibility for laboratory support, including training, quality assurance, and quality control, falls under the domain of the NCAIDS National Reference Laboratory. Since 2004, local CDCs and hospitals in high prevalence areas have become well equipped with flow cytometry capacity, such as Becton Dickinson FACSCount machines, and the capacity to conduct routine blood and biochemistry testing.

\section{Database}

To improve the three month data collection system employed in the initiation phase of the Free ART Program, information on patients is now collected through the DataFax system. This system uses paper clinical records, which are kept at the service delivery site, but which are faxed to the National Center for Public Health Surveillance and Information Services, Chinese CDC, and automatically converted into an electronic database with data verification and cleaning capabilities. Information is collected at treatment initiation, each follow-up visit, regimen change, and treatment termination. Demographic information, laboratory test results, clinical signs and symptoms, and self-reported adherence are collected. With 
the exception of Henan province, which uses its own electronic medical record system, this real time data collection system is being used by all regions providing free ART.

\section{Special populations}

The government's initial provision of free ART was an emergency response to treat the large numbers of former blood and plasma sellers developing AIDS in central China. To date, the majority of patients receiving treatment are still adults who were former plasma and blood sellers despite the fact that the greatest percentage of HIV infected patients are intravenous drug users (IDUs) and that there is an increase in the prevalence of HIV positive children in recent years. For a variety of reasons, IDU treatment is very challenging. Most IDUs are marginalized and mobile, without regular access to medical care, which decreases adherence rates and leads to drug resistance. The first treatment site aimed at IDUs was initiated in early 2005 in Sichuan province. A dual strategy of linking ARV treatment with methadone maintenance services and of providing DOT was adopted for this site. As China's methadone program expands, linkages will increase with HIV/ AIDS treatment and care services and increasing numbers of IDUs will be able to access free ART.

HIV positive patients co-infected with tuberculosis and hepatitis form another special population. High rates of co-infection are a unique characteristic of China's epidemic and create a unique challenge. The National TB Center and international organizations are spearheading the initiative to address TB/HIV co-infection issues.

Before June 2005, pediatric ARV formulations were not available in China. In June, a one year supply of pediatric ARV and cotrimoxazole formulations otherwise unavailable in the Chinese market became available, through a Clinton Foundation donation, for 200 children who were identified as needing treatment,. Counties in six provinces were selected on the basis of prevalence and experience to form the initial pediatric treatment sites. Numerous trainings for physicians, nurses, laboratory technicians, and treatment support providers were conducted with assistance from domestic and international experts. In order to create sustainability, a great deal of effort is going into ensuring the continuation of the pediatric drug supply, capturing a better picture of the patient population, and providing family education and supportive services.

\section{Operational research}

Operational research is being conducted to improve the clinical and programmatic aspects of the Free ART Program. On-going research includes efforts to compare and evaluate different ART delivery models in resource- limited settings and a prospective observational cohort study of children examining tolerance and adherence to ART the effects of ART on disease progression, HIV/AIDS related conditions, child development, and health system utilization. Several ongoing studies examine the effects of behavioral interventions including a study comparing the effectiveness of DOT in resource-limited primary care versus tertiary care settings, a development project to design and test culturally appropriate and feasible adherence programs, a prospective observational cohort study to understand the risks and benefits, as well as adherence, of cotrimoxazole prophylaxis for Chinese patients with AIDS, a randomized comparison of adherence of ART in IDUs receiving DOT versus standard drug delivery, and a prospective observational study of adherence among IDUs receiving ART in the context of methadone maintenance therapy. Studies of resistance, focusing on non-subtype B strains of the virus, are also on-going. Partners for research include various hospitals around China, international organizations such as the WHO, and U.S. universities. Support for operational research comes from the $\mathrm{MOH}$, the Ministry of Science and Technology as well as the U. S. National Institutes of Health.

\section{DISCUSSION}

The effort to provide nationwide access to free high quality treatment and care is hampered by a set of underlying challenges and a set of technical issues. The underlying challenges are problems that need to be addressed, but they are also barriers preventing the resolution of the technical issues that China faces. China's best resource in addressing both types of challenges is government leadership and support. Over the past few years, the central government has made great strides in the fight against HIV/ AIDS, and combating the disease is clearly a high priority. However, China is a large and complex country, and it will take the efforts of all sectors and levels of government and society to adequately address the country's epidemic, including the provision of free ART.

The underlying challenges faced by China revolve around the country's health system and its patient population. With decreases in government funding for health service providers and an underdeveloped medical insurance system, most Chinese patients receive care through out-of-pocket payments. The lower the level of the hospital, for example a county hospital as opposed to a provincial hospital, the less funding it receives from the government and the more it must extract from the patient in order to stay open. Physicians must generate revenue for the hospital, and laboratory tests and prescriptions are often the method used for revenue generation. In addition to this incentive structure, the lower the level of the hospital, the fewer resources, 
both capital and human, it has. In China, many country physicians, not to mention township and village physicians, do not have a M.D. In addition, lower level hospitals often lack basic equipment, including the tools needed to ensure universal precautions and infection control such as disposable gloves and needles.

The vast majority of people living with HIV/AIDS (PLWHA) are poor, live in rural areas, have little education, and due to changes in economic structure, faces pressures to migrate in search of employment. Often, they are marginalized from their communities either due to prior behavior, such as drug use, or after infection, due to stigma and discrimination. In order to give these patients access to the benefits of ART, the government mandated the "Four Free and One Care" policy and the subsequent implementation of the Free ART Program. The government has committed itself to provide free drugs, free testing, free care, and free supportive services based on the principle of community and home-based treatment to increase accessibility of services to China's patient population. However, given the incentive structure of the health system, the lack of resources within the system, especially at the lower levels, stigma, discrimination, fear of contracting the virus, both on the part of the general public and health workers, low levels of patient education, and the pressure on patients to migrate for work, the ability to provide treatment and care becomes extremely difficult.

Rural health reform is a priority of the Chinese government, but improvements are difficult and will take time. To mitigate the effects of these underlying challenges for HIV/AIDS patients, the government and other organizations have implemented innovations such as voucher systems for hospitals, rewards for physicians who treat HIV/AIDS, incentives for well trained physicians from urban areas to treat rural patients on a rotational basis, free machinery and reagents for hospitals providing care, an emphasis on training both health workers and patients, linkages to outreach programs, and general education to decrease stigma and discrimination. In addition, work is being done to increase the number of horizontal linkages between HIV/AIDS program and other programs such as $\mathrm{TB}$, maternal and child health, and rural insurance schemes.

While work to alleviate underlying challenges is underway, these challenges still create an environment in which it is more difficult to resolve technical issues that would improve the Free ART Program. These technical issues relate to implementation, human capacity, and access.

Policies and programs are difficult to implement because a large variety of actors, both domestic and international, are involved. Coordination and communication is crucial to making full use of diverse resources; however, given the large volume of work and human resource shortages, coordination and communication are often neglected. In addition, China has yet to implement a comprehensive monitoring and evaluation (M\&E) system, which is vital for effective and efficient implementation.

On the issue of human capacity, even though large numbers of health workers have received training, the gap between gaining knowledge and applying knowledge can only be bridged with time and the accumulation of experience. However, there is not only a lack of clinical capacity, but also a lack of management capacity. In recent years, increasing attention on HIV/AIDS has fortunately led to increases in funding and programs. However, the corollary increase in the quantity and quality of personnel has been insufficient. There are not enough people to manage the large work load, and many people lack management expertise.

The third technical issue deals with access to drugs and equipments. Even though there are over twenty types of ARVs in the world, only seven are available in China. China has developed a CD4 network and has equipped treatment centers with the machinery needed for routine blood and biochemistry tests, but viral load testing and resistance testing are not free and are often unavailable for patients due to cost.

Much work has also been done to address the technical issues China facing. In order to improve coordination and communication, offices within the MOH and NCAIDS have streamlined their operations. For example, international organizations working on HIV/AIDS are now solely managed through the MOH Department of International Cooperation. The MOH and NCAIDS are in the process of developing a comprehensive M\&E framework, and the treatment and care component of this system is entering the pilot phase. Increases in funding have gone to hire new personnel, and training courses are being designed to address weaknesses in management. The government is working with domestic and international partners to make more ARVs accessible and to decrease the cost of ARVs and testing.

Despite the challenges that China faces, it has come a long way in its fight against HIV/AIDS, and especially in the realm of treatment and care. China's example shows that while it is extremely difficult to provide treatment and care in a rural, resource-limited setting, more and more people are accessing treatment and the Free ART Program has saved thousands of lives. A great deal of progress has been made despite numerous challenges, and in China, this has been made possible by the attention, support, and leadership of the government. In addition, China has been fortunate to receive a great deal of assistance from international organizations, ranging from UN organizations to 
foundations to private enterprises, which provide financial support and more importantly their technical resources and experience. In a country of its size and diversity, challenges will continue to emerge, but with determination and a diverse set of resources, China will continue to progress toward its goal of providing nationwide access to high quality treatment and care, and hopefully in the process, provide valuable lessons for other developing countries.

\section{REFERENCES}

1 China Ministry of Health and UN Theme Group on HIV/AIDS in China, A Joint Assessment of HIV/AIDS Prevention, Treatment and Care in China, Beijing: Ministry of Health, 2003:1.

2 Wang LD. HIV/AIDS Prevention and Control in China, Ministry of Health and Global Business Coalition on HIV/AIDS Joint Summit on Business and AIDS in China, 2005.
3 China Global Fund 3 Proposal Working Group, China Global Fund Round 3 Proposal HIV/AIDS Component - China CARES (China Comprehensive Aids RESponse): A Community-Based HIV Treatment, Care and Prevention Program in Central China, Unpublished, 2003.

4 Measures for Management of Drug Treatment of HIV/AIDS and Common OIs at No/Reduced Charge, 2004, Ministry of Health and Ministry of Finance (Wei Ji Kong Fa) No. 107.

5 Opinions Concerning the Management of HIV Antiviral Treatment, 2004. Ministry of Health and State Administration for Traditional Chinese Medicine (Yi Fa WEI) No. 106.

6 National Center for STD/AID Prevention and Control. National ARV Treatment Situation Report. Unpublished, 2005: 1.

7 National Center for STD/AIDS Prevention and Control, DataFax Report. Unpublished, 2005: 1-2.

8 Chinese Center for Disease Control and Prevention, China Free ART Manual, Beijing: Ministry of Health 2005:7-8. 